\title{
Control of microtubule dynamics by the antagonistic activities of XMAP215 and XKCM1 in Xenopus egg extracts
}

Régis Tournebize* $\dagger$, Andrei Popov*, Kazuhisa Kinoshita *, Anthony J. Ashford*, Sonja Rybina *, Andrei Pozniakovsky*, Thomas U. Mayer* $\ddagger$, Claire E. Walczak§, Eric Karsenti ${ }^{*}$ and Anthony A. Hyman* $q$

${ }^{*}$ Cell Biology Program, EMBL, Meyerhofstrasse 1, 69117 Heidelberg, Germany tPresent address: Pathogénie Microbienne Moléculaire, Institut Pasteur, 28 rue du Dr Roux, 75724 Paris Cedex 15, France $\ddagger$ Present address: Harvard Medical School, Department of Cell Biology, 250 Longwood Avenue, Boston, Massachusetts 02115, USA $\$$ Medical Sciences Program, Indiana University, Jordan Hall 306, 3rd and Hawthorne, Bloomington, Indiana 47405, USA ge-snail: hyman@embl-heidelberg.de

Microtubules are dynamic polymers that move stochastically between periods of growth and shrinkage, a property known as dynamic instability. Here, to investigate the mechanisms regulating microtubule dynamics in Xenopus egg extracts, we have cloned the complementary DNA encoding the microtubule-associated protein XMAP215 and investigated the function of the XMAP215 protein. Immunodepletion of XMAP215 indicated that it is a major microtubule-stabilizing factor in Xenopus egg extracts. During interphase, XMAP215 stabilizes microtubules primarily by opposing the activity of the destabilizing factor XKCM1, a member of the kinesin superfamily. These results indicate that microtubule dynamics in Xenopus egg extracts are regulated by a balance between a stabilizing factor, XMAP215, and a destabilizing factor, XKCM1.

\begin{abstract}
1. during cell division and differentiation. Despite the importance of these changes, little is known about how they are controlled. Microtubules exist in two states, either growing or shrinking, and they transit stochastically between these two states, a behaviour known as dynamic instability'. The transition from growth to shrinkage is called a catastrophe, and the transition from shrinkage to growth, a rescue. Thus, microtubule behaviour can be
\end{abstract}

described by four parameters, namely the growth rate, the shrinkage rate, the catastrophe frequency, and the rescue frequency $y^{2,3}$. In vitro, in pure tubulin solutions, two of these parameters (growth rate and catastrophe rate) are tightly linked and strictly determined by the free tubulin concentration ${ }^{2}$. In contrast, the different parameters of microtubule dynamics are regulated independently in vivo, indicating that the combinatorial action of several regulatory factors may determine the values of each of these parameters and the a

\begin{tabular}{|c|c|c|c|}
\hline MKL & PIDQKCEHKV & WKARLNGYEE & AVKLFQKIVD \\
\hline AALVYVENAH & VAGKTTGEVV & NGVVNNKVFNQ & PKARAKELGA \\
\hline WACVETVRK & ALSEFGSKIM & TLKPIIKVLP & KLFESREKAI \\
\hline LKELEEEWVK & LPQSAPKQTR & FLRSQQDLKA & KFBQQQAAGD \\
\hline KDEYDKIEAK & KWQERKEALE & AVEALVKNPK & IEAGDFADLV \\
\hline RKKEGSYAGH & IVPTILEKFK & EKKPQVVQAL & QEAIDAVFLT \\
\hline RSFRHCTPST & LPKSLLKPEC & VALEKOINDS & APEVRDAAFE \\
\hline ECADKAELAN & GKKGGAAAGE & KKETKAPAAA & PGKPVPNQGA \\
\hline PASSGATAKG & KKAVENKEII & EQELSPEACE & BRAAAVLPAS \\
\hline DIPCQALVKM & LAKKPGEKET & NFQVMQMKS H & IVALIAQKGN \\
\hline SGIAEACTLP & WTAEQVVSLA & FAQKNDKNQS & ETLNWLSNAI \\
\hline SAITLLGVMY & LYMGAPLRMF & FEEEKPALLS & QIDAEFEKMK \\
\hline PADVTDLLPR & TDISDKISSD & LVSKIEDKNW & KIRKEGLDEV \\
\hline ILVQQTLTIS & QQLSTAMGHN & IKQHVKNLGM & PI ITVLGDSK \\
\hline EELKKENPFL & RQELLGWLAE & KLPSMRTVPS & DLQLCVPYLY \\
\hline SKATSKLKPA & SKDQVVALLE & KAKASMPAKP & AGPPGKASSK \\
\hline PKKTKPGTQA & SKAKTQEYSS & aneses? & 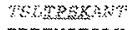 \\
\hline GPIYIIVPNG & KEQRVKDEKA & LKVLKUNETT & FRDEYIEQLK \\
\hline TEHLESEKEG & VISCLDLVLK & WFTLRFFDTN & TSVLMKCLEY \\
\hline VGEPKDIVRK & DVRAILTKMC & QVYDASKMFN & FVMEGTKSKN \\
\hline JKEIAIHIGD & RDTTVVRNAAL & NTIVTVYNVH & GEQVFKLIGN \\
\hline EKPQRVQSAN & ASILRKAPPE & DMSSKLNOAR & NMGGHTEPSH \\
\hline KLDEIFEPVL & IPEPKIRAVS & PHFDDMHSSNT & ASTINFVISQ \\
\hline GHIDQFLIAT & FMQLRLA YNT & HMADERLDKD & DIVRLYSCII \\
\hline LMLDARIEDL & EEGQQVVRSV & NLLVVKVULEK & SDQTNIISAL \\
\hline LLPEA INNLN & LDRILLD $1 H N$ & FMRVLPKEKL & KQHKSEMPMR \\
\hline ELEAHLLRVM & KHSIDRTGSK & GDKETEKGAS & CIEDKVGKAN \\
\hline KYSDADIKPF & LKNSSQFFQS & YVERGLRLIE & MEREGKARIA \\
\hline EEVGPSVYLE & RLKILRQRCG & LDNAKQDERP & PLTSLLSKSS \\
\hline & & KKRLERI & \\
\hline
\end{tabular}

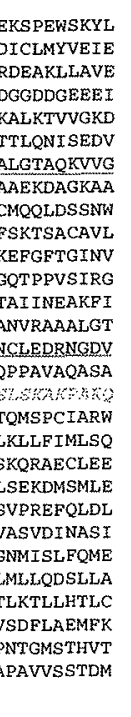

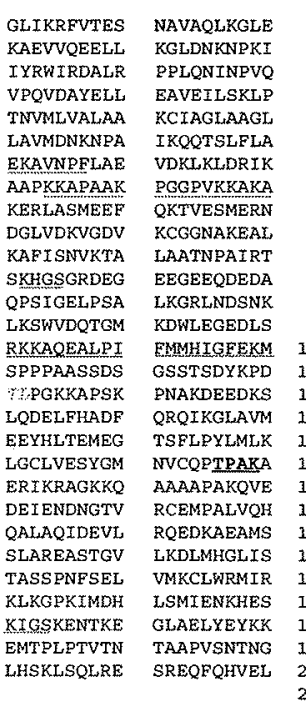

b
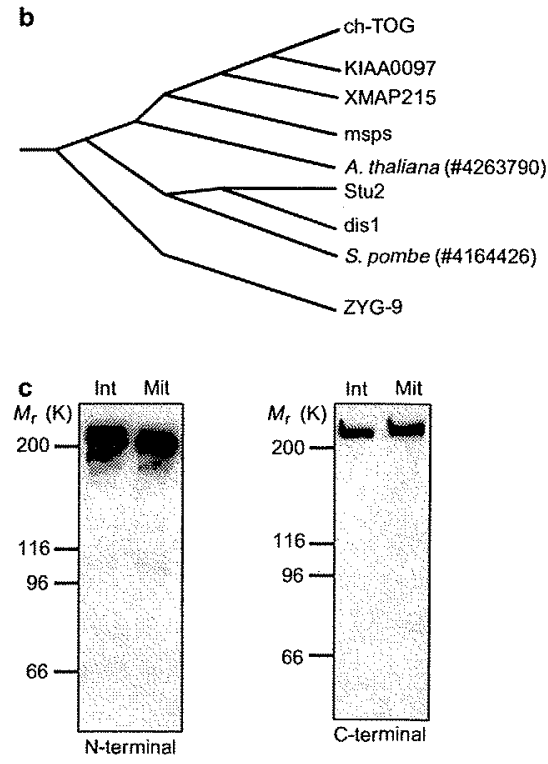

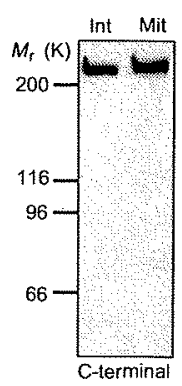

Figure 1 XMAP215 amino-acid sequence and homology to other members of the protein family to which it belongs. a, Amino-acid sequence of XMAP215. Red underlining, predicted sites of phosphorylation by the kinase MARK; green underlining, the bipartite nuclear-localization signal; blue underlining, HEAT repeats; red italics, the insertion found in XMAP215 that is absent in ch-TOG/KIAA0097; bold underlining, a putative site of phosphorylation by $\mathrm{Cdc} 2 . \mathbf{b}$, Phylogenetic tree of XMAP215 relatives. msps, Mini spindles proteins. $c$, Specificity of antich-TOG antibodies. Immunoblots done using $80 \mu \mathrm{g}$ interphasic (Int) and mitotic (Mit) Xenopus egg extracts show that affinity-purified antibodies raised against the $N$ and $C$ termini of ch-TOG recognize a single protein of $M_{1}, 215 \mathrm{~K}$. 

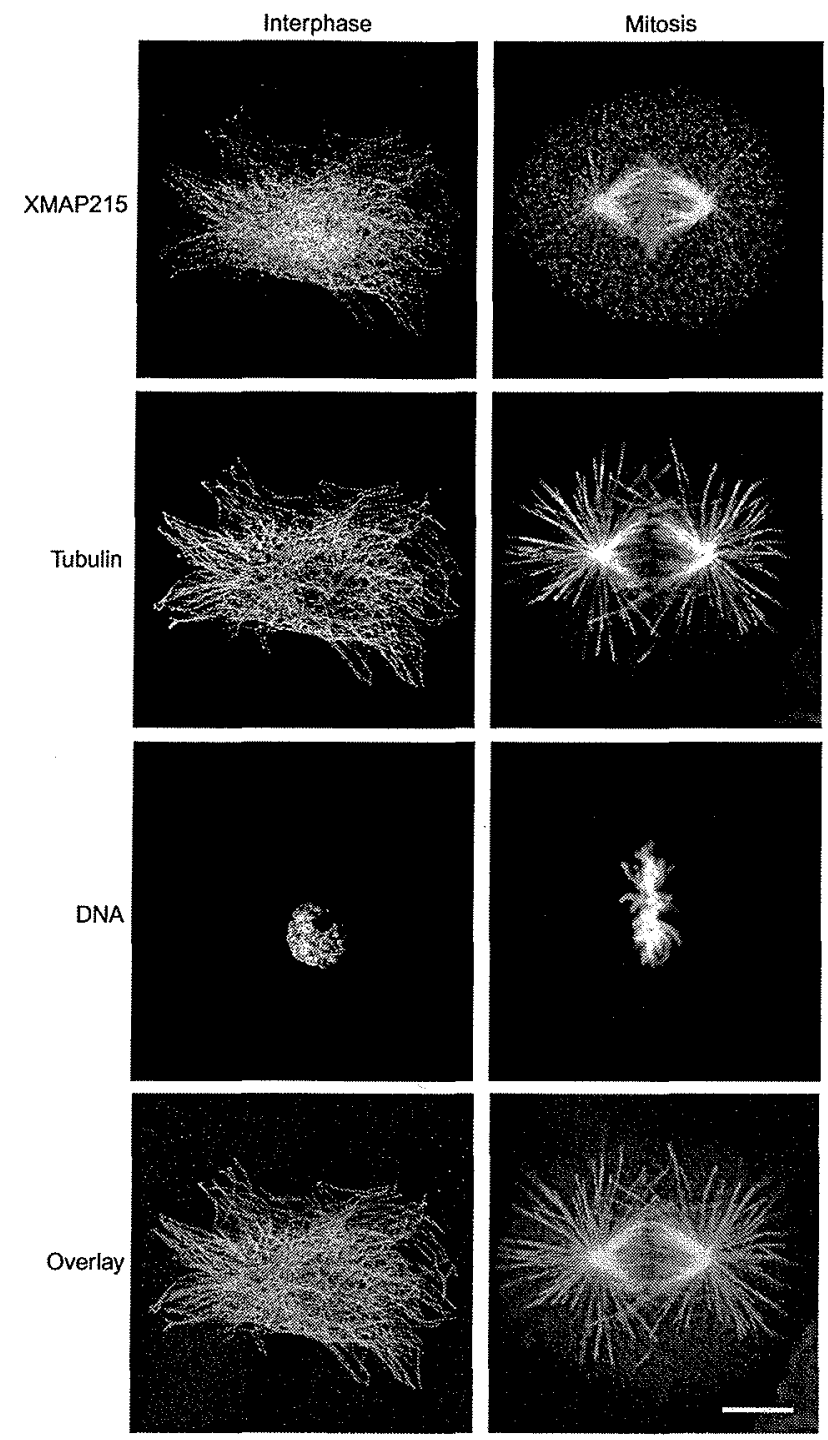

Figure 2 Immunolocalization of XMAP215 protein in XL177 cells. An interphasic cell is shown on the left, and a mitotic cell is shown on the right. In the overlay, microtubules are green, XMAP215 is red, and DNA is blue. Scale bar represents $10 \mu \mathrm{m}$.

resulting properties of the microtubule arrays $\mathrm{s}^{2,4}$.

Various microtubule-regulating factors have been identified in Xenopus laevis egg extracts. One of these, XMAP215, has the unusual ability to increase both growth and shrinkage rates without affecting the rescue and catastrophe frequencies ${ }^{5,6}$. In contrast, XMAP230 mostly reduces the catastrophe frequency ${ }^{7}$ whereas XMAP310 increases the rescue frequency. Two factors that induce catastrophes, albeit by different mechanisms in the presence of pure tubulin, are Stathmin/Op18, a heat-stable protein of relative molecular mass $18,000\left(M_{\mathrm{r}} 18 \mathrm{~K}\right)$, and XKCMl, a member of the kinesin superfamily $y^{y-13}$. Although the identification of these factors represents significant progress, our understanding of the regulation of microtubule dynamics in vivo is still fragmentary. Control of microtubule dynamics is likely to involve a combination of destabilizing and stabilizing factors. The challenge is to identify these important factors and to understand how modulation of one factor in the context of the other factors may be involved in changing the length dis-
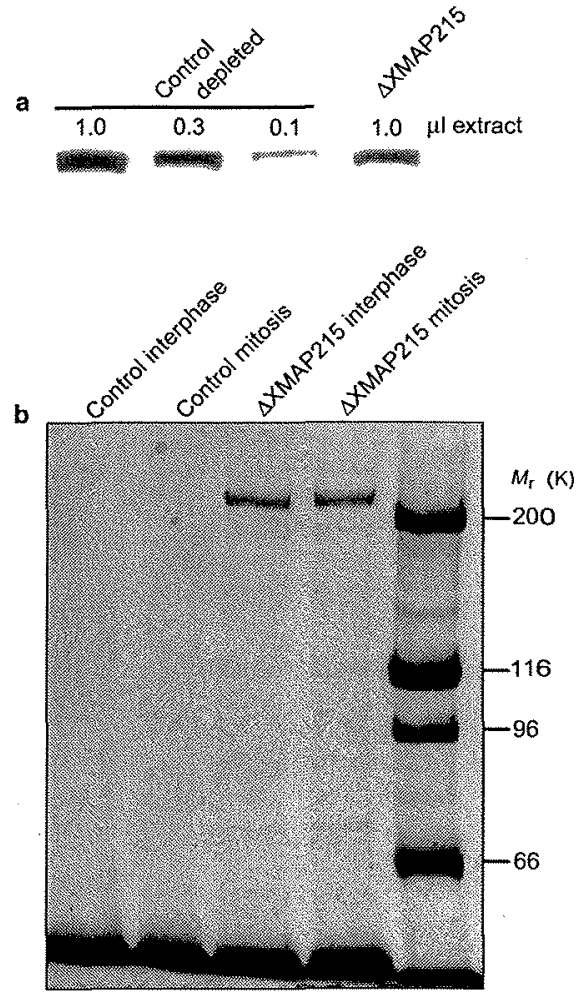

Figure 3 XMAP215 depletion from Xenopus egg extracts. a, Immunoblot showing the depletion efficiency. $1 \mu \mathrm{l}, 0.3 \mu \mathrm{l}$ and $0.1 \mu \mathrm{I}$ of a control depleted Xenopus egg extract and $1 \mu$ l of a XMAP215-depleted extract were run on a gel and XMAP215 was revealed with an antibody against the ch-TOG $\mathrm{C}$ terminus. The amount of XMAP215 remaining in the XMAP215-depleted extract corresponds to $30 \%$ of the amount found in the control depleted extract. $\mathbf{b}$, Coomassie-blue-stained gel of the XMAP215 immunoprecipitates from interphase and mitotic extracts. Only a band of $M_{t} 215 K_{t}$ corresponding to XMAP215, was immunoprecipitated in both interphase and mitotic extracts. The bands at the bottom correspond to the IgG heavy chains.

tribution and dynamics of microtubule arrays.

Among the microtubule-associated proteins (MAPs) characterised to date, XMAP215 is of particular interest because, in interphase, it stimulates the growth rate of microtubules at their plus (fast growing) ends ${ }^{14}$ without affecting the catastrophe frequency ${ }^{5,6}$. Furthermore, XMAP215 is a member of a family of proteins that seems to be involved in the control of microtubule organization. This family includes a human homologue, ch-TOG ${ }^{15}$, the Mini spindles proteins in Drosophila melanogaster ${ }^{16}$, ZYG-9 in Caenorhabditis elegans ${ }^{17}$, Stu 2 in Saccharomyces cerevisiae ${ }^{18}$ and p93 ${ }^{\text {Dist } 1}$ in Schizosaccharomyces pombe ${ }^{19-21}$. We thus investigated the function of XMAP2 15 in control of microtubule dynamics in Xenopus egg extracts, and found that XMAP215 strongly modulates the catastrophe frequency by opposing the microtubule-destabilizing activity of XKCM1.

\section{Results}

Molecular cloning of XMAP215. To investigate the in vivo function of XMAP215, we cloned and sequenced the corresponding CDNA, which encodes a protein of 2,065 residues (Fig. 1a). The XMAP215 amino-acid sequence is highly similar to those of the human proteins ch-TOG ${ }^{22}$ (78.4\% identity) and KIAA0097 (ref. 23; 79\% identity). Ch-TOG and KIAA0097 are identical with the exception of a 60 -amino-acid insertion at position 1,564 of ch-TOG, also present 


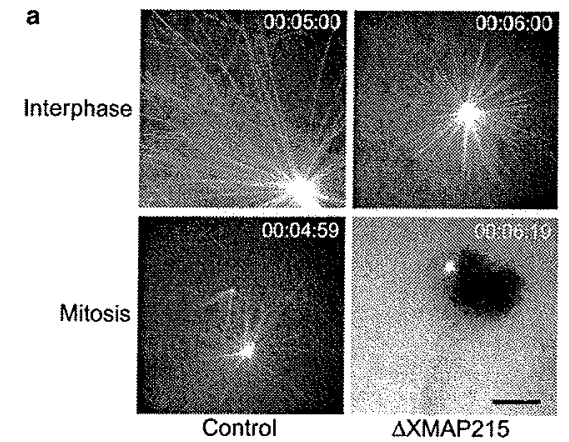

b
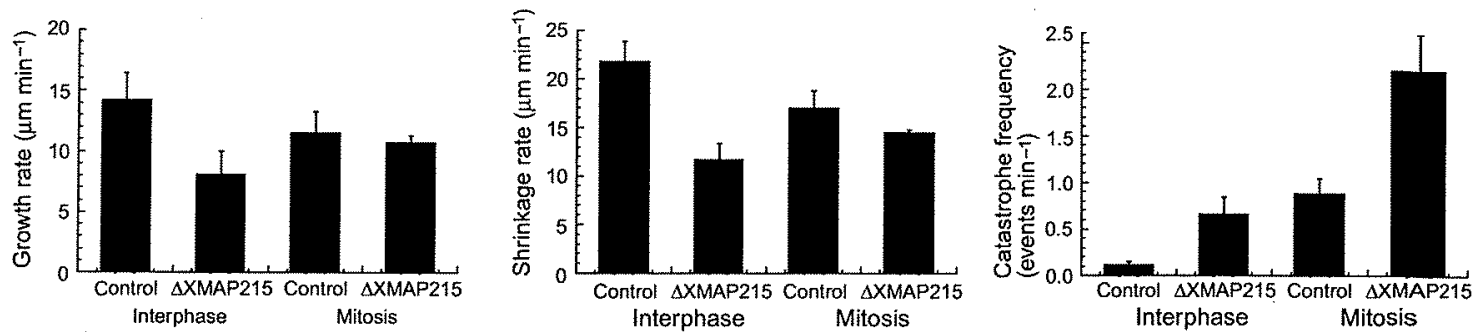

Figure 4 XMAP215 is a major regulator of microtubule dynamics. a, Images of a microtubule aster as observed during recording of movies. Microtubules in interphase and mitotic control depleted extracts have a normal size after $5 \mathrm{~min}$ of recording. However, in interphasic XMAP215-depleted extracts, microtubules are shorter than those in control extracts after $6 \mathrm{~min}$ of recording. Microtubules are barely visible in mitotic XMAP215-depleted extracts, such that images had to be recorded for $15 \mathrm{~min}$ instead of $5 \mathrm{~min}$. Scale bar represents $10 \mu \mathrm{m}$. $\mathrm{b}$, Quantification of microtubule dynamics. In comparison with controls, the growth rate and the shrinkage rate decrease after XMAP215 depletion in interphase extracts but not in mitotic extracts. The catastrophe frequency is increased in both interphase and mitotic XMAP215-depleted extracts relative to controls. Data shown are means \pm s.e.m. in XMAP215, and two amino-acid changes from alanine to glutamate at positions 1,814 and 1,822 in the KIAA0097 sequence. XMAP215 has another 36-amino-acid insertion at position 1,140 of ch-TOG. XMAP215 is a member of a protein family that includes p93 ${ }^{\text {Disl }}$ and a putative 809-amino-acid protein (GenBank accession number 4164426) from $S$. pombe, Stu2 from $S$. cerevisiae, ZYG-9 from C. elegans, Mini spindles from D. melanogaster and a putative 2,021-amino-acid protein from Arabidopsis thaliana (GenBank accession number 4263790) (Fig. 1b).

Analysis of the XMAP215 amino-acid sequence showed that it contains two putative sites of phosphorylation by the kinase Cdc2. One is located in the 36-amino-acid insertion (position 1,154) and the other is at position 1,396. XMAP215 also contains two bipartite nuclear-localization signals (positions 544-561 and 556-573) and two HEAT repeats at positions 436-477 and 1,015-1,052 (ref. 24) Interestingly, the amino-acid motif KXGS, which is the target for the kinase MARK $^{25}$ and is found in the microtubule-binding domains of the MAPs Tau, MAP4 and MAP2B ${ }^{26-29}$, is present once in ch-TOG/KIAA0097 and three times in XMAP215 (Fig. 1a).

XMAP215 localizes to microtubules in interphase and mitosis. To attempt to understand the function of XMAP215 in vivo, we raised antibodies against the amino and carboxy termini of XMAP215 and KIAA0097/ch-TOG. In interphase and mitotic Xenopus egg extracts, the antibodies raised against both the $\mathrm{N}$ and the $\mathrm{C}$ terminus of ch-TOG recognized a single band with an $M_{\mathrm{r}}$ value of about $215 \mathrm{~K}$ (Fig. 1c). Identical results were obtained with the antiXMAP215 antibodies (data not shown). Thus, antibodies to both ch-TOG and XMAP215 recognize XMAP215 in Xenopus egg extracts.

We next determined the localization of XMAP215 in XL177 Xenopus tissue culture cells (Fig. 2). Cells were fixed and stained with antibodies against the $\mathrm{N}$ terminus of XMAP215. In interphase, XMAP215 co-localized with microtubules. During mitosis, the spindle and the centrosomes were brightly stained whereas astral microtubules were poorly labelled. Similar results were obtained with antibodies to the $\mathrm{N}$ terminus of ch-TOG. Thus, XMAP215 has an intracellular distribution that suggests an involvement in the control of microtubule dynamics.

XMAP215 regulates parameters of microtubule dynamics in extracts. To attempt to understand the role of XMAP215 in the control of microtubule dynamics, we used antibodies specific for the $\mathrm{C}$ terminus of ch-TOG to deplete Xenopus egg extracts. As shown in Fig. 3a, 30\% of XMAP215 remained in the extract following depletion. Routinely, between $50 \%$ and $70 \%$ of XMAP 215 was depleted from the extract. In both interphase and mitotic extracts, a single band of $M_{\mathrm{r}} \sim 215 \mathrm{~K}$ was precipitated by the antibody against the ch-TOG C terminus (Fig. 3b). Furthermore, the protein precipitated using antibodies directed against the $C$ terminus of XMAP215 was recognized by anti-chTOG antibodies, showing that both antibodies recognized the same protein (data not shown). These observations indicate that the depletion was specific to XMAP215.

To assay microtubule dynamics, we observed individual microtubules by fluorescence video microscopy of extracts depleted of XMAP215. In both interphasic and mitotic extracts, the depletion of $\sim 60 \%$ of XMAP 215 was enough to result in much shorter and more dynamic microtubules (Fig. 4a). In interphase, the growth and the shrinkage rates decreased from $14.2 \mu \mathrm{m} \mathrm{min}^{-1}$ to $8.0 \mu \mathrm{m}$ $\mathrm{min}^{-1}$ and from $21.7 \mu \mathrm{m} \mathrm{min}^{-1}$ to $11.6 \mu \mathrm{m} \mathrm{min}^{-1}$, respectively (Fig. $4 b$ ). This observation correlates well with what is known about the effect of XMAP215 on pure tubulin ${ }^{5,6}$. To our surprise, we also observed an increase in the interphase catastrophe frequency, from 0.1 events $\mathrm{min}^{-1}$ to 0.7 events $\mathrm{min}^{-1}$ (similar to that observed in mitosis). In mitotic extracts, the effect was even more dramatic, as microtubules were often not observed during the first $15 \mathrm{~min}$ of recording in XMAP215-depleted extracts ( $5 \mathrm{~min}$ is normally long enough to record microtubule dynamics). The growth and shrinkage rates in mitotic extracts were not affected by XMAP215 deple- 


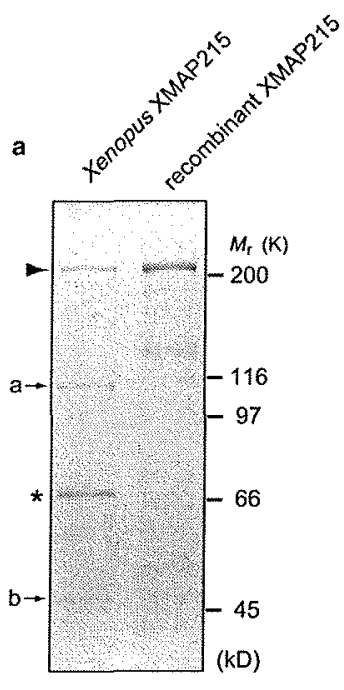

b

Xenopus XMAP215 Recombinant XMAP215 add back add back
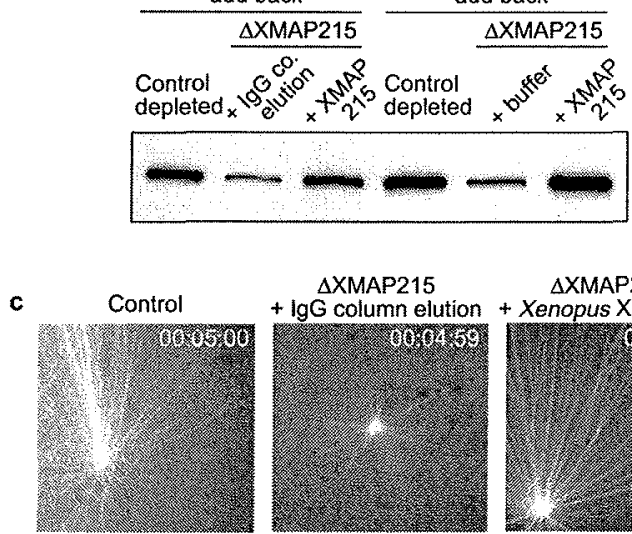

$\triangle X M A P 215$

$\triangle X M A P 215$
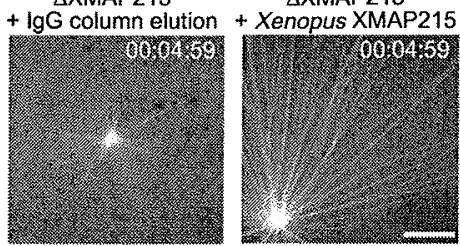

c
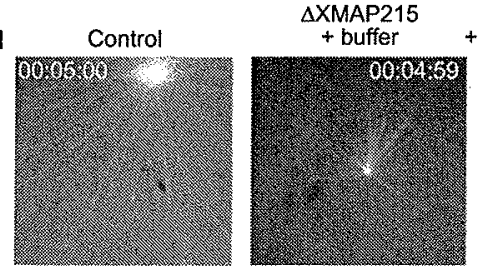

$\triangle X M A P 215$ recombinant XMAP215
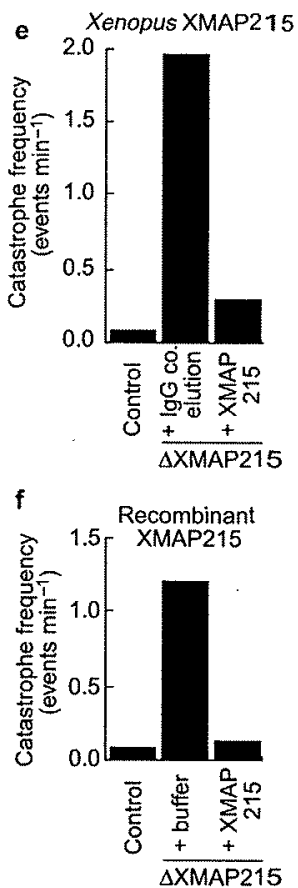

Figure 5 Readdition of purified XMAP215 to depleted extracts. a, Coomassieblue-stained SDS get showing position of XMAP215. Left, Xenopus XMAP215 was purified on an affinity column of antibodies directed towards peptides from the XMAP215 C terminus. The asterisk shows the position of BSA contained in the elution buffer. The associated protein indicated by arrow ' $b$ ' is also seen in immuno-affinity purification with control lgG. Variable amounts of another protein, indicated by arrow ' $a$ ', were analysed by mass spectrometry and identified as vitellogenin-A2 precursor (Swiss-plot accession number P18709), a common contaminating protein. Right, recombinant XMAP215 was expressed in insect cells and purified on a Mono-S column. b. Immunoblot of immunodepleted extracts before and after adding back XMAP215. Interphase extracts were immunodepleted with control IgG (lanes 1,4) or
anti-ch-TOG antibody (lanes 2, 3, 5, 6). An elution fraction from the IgG column (lane 2), Xenopus XMAP215 (lane 3), control buffer (lane 5) or recombinant XMAP215 (lane 6) was then added to XMAP215-depleted extracts. c, d, Images of microtubule asters in control depleted extracts (left), and XMAP215-depleted extracts before (middle) and after (right) adding back immunopurified (c) or recombinant (d) XMAP215, respectively. Both purified proteins rescued the aster size of XMAP215 depleted extracts. Scale bar represents $10 \mu \mathrm{m} . \mathrm{e}, \mathrm{f}$, Effects of addition of immunopurified and recombinant XMAP215 on the catastrophe frequency in XMAP215-depleted extracts. Adding back purified proteins caused a 7-10-fold suppression of the catastrophe frequency observed in XMAP215-depleted extracts. tion, being respectively $11.5 \mu \mathrm{mmin}^{-1}$ and $16.9 \mu \mathrm{m} \mathrm{min}^{-1}$ in control depleted extracts and $10.6 \mu \mathrm{m} \mathrm{min}^{-1}$ and $14.4 \mu \mathrm{m} \mathrm{min}^{-1}$ in XMAP215-depleted extracts. However, the catastrophe frequency increased from 0.9 events $\mathrm{min}^{-1}$ to 2.1 events $\mathrm{min}^{-1}$ (Fig. $4 \mathrm{~b}$ ).

To confirm that the effects observed were due to XMAP215 removal, we added either immunopurified XMAP215 or XMAP215 produced in baculovirus-infected cells back to the depleted extracts (Fig. 5a). Restoration of the endogenous level of XMAP215 with either protein (Fig. $5 \mathrm{~b}$ ) allowed microtubules to grow long again (Fig. 5c, d), showing that XMAP215 is sufficient to rescue the observed phenotype resulting from the earlier depletion of XMAP215. We next analysed the parameters of microtubule dynamics in these conditions. The catastrophe frequency decreased dramatically to levels similar to that observed in control depleted extracts (Fig. 5e, f), showing that XMAP215 is a major regulator of catastrophes in Xenopus egg extracts. We also analysed the growth and shrinkage rates. Both parameters were increased to some extent. Nevertheless, this change was variable and never reached control levels (data not shown). A possible explanation for this discrepancy is that our purified proteins were not fully active in rescuing the phenotype of the growth and shrinkage rates. However, the main phenotypes characterized, such as the change in microtubule length and in the catastrophe frequency, are fully reverted by the addition of purified XMAP215. Together, these observations indicate that, first, XMAP215 is required for at least half of the growth and the shrinkage rates in interphase but does not contribute to these parameters during mitosis; second, catastrophe factors are also active in interphase; and third, XMAP215 antagonizes the function of catastrophe factors both in interphase and in mitosis. XMAP215 and XKCM1 act antagonistically. We wanted to know which catastrophe factors oppose XMAP215 activity. Depletion of one such factor, Stathmin/Op18, decreases the catastrophe rate about twofold in mitosis ${ }^{30}$. To assess the degree to which another catastrophe factor, $\mathrm{XKCM1}$, was responsible for the catastrophe rate in mitosis, we added inhibitory anti-XKCM1 antibodies ${ }^{10}$ to mitotic extracts and determined the parameters of microtubule dynamics (Fig. 6b). After addition of anti-XKCMI antibodies to mitotic extracts, the catastrophe frequency declined sevenfold. We conclude that XKCM1 is the major factor regulating the catastrophe rate in mitotic extracts. To determine whether XKCMI was also responsible for catastrophes in interphase, we studied microtubule dynamics after addition of anti-XKCMl inhibitory antibodies to interphase and mitotic extracts depleted of XMAP215. This procedure resulted in an increased length of the microtubules in both interphase and mitotic extracts (Fig. 6a). We then quantified the different parameters of microtubule dynamics. In four different depletions of XMAP215 from interphase and mitotic extracts, we observed an increase in the catastrophe frequency, followed by a decrease after inhibition of XKCM1. Values from two similar experiments are shown in Fig. 6b. The catastrophe frequency in an interphase extract increased from 0.1 events per minute to 0.7 events min $^{-1}$ after depletion of XMAP215, and was reduced to 0.2 

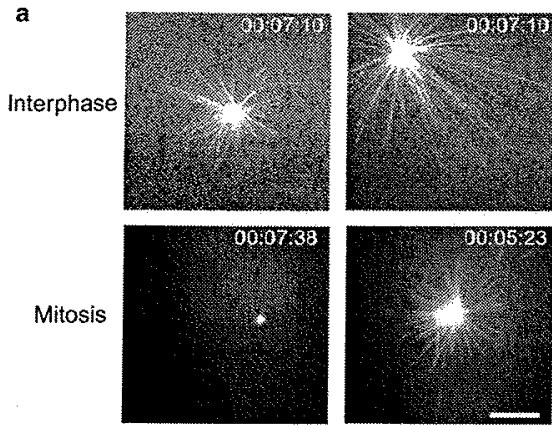

$\triangle \mathrm{XMAP215}$

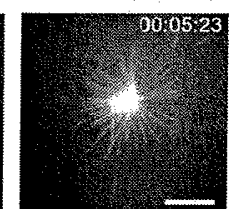

$\triangle \mathrm{XMAP} 215$ + anti-XKCM antibody

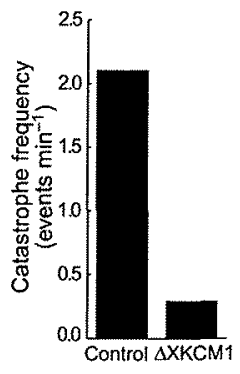

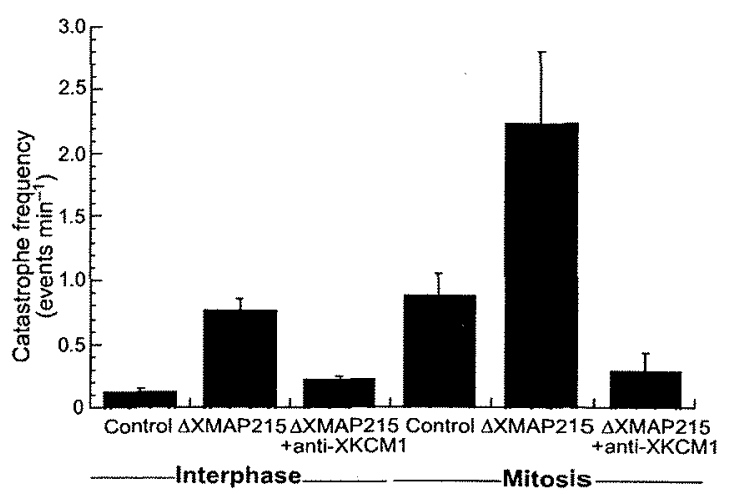

Figure 6 Inhibition of XKCM1 partially rescues the effect of XMAP215 depletion on the catastrophe frequency. $a$, Images of microtubule asters in XMAP215-depleted extracts before and after inhibition of XKCM1. Microtubules grow longer after XKCM1 inhibition. $b$, Left, change in catastrophe frequency after inhibition of XKCM1 in mitotic extracts. Right, quantification of microtubule dynamics in XMAP215-depleted extracts after XKCM1 inhibition. Only a change in the catastrophe frequency was observed; the growth and shrinkage rates were similar to those shown in Fig. 4 and are not shown here. Inhibition of XKCM1 decreases the catastrophe frequency in both interphase and mitotic extracts. Data are shown as means \pm s.e.m. events $\min ^{-1}$ after addition of anti-XKCM1 antibodies. In a mitotic extract depleted of XMAP215, addition of anti-XKCM1 antibodies decreased the catastrophe frequency from 2.2 events $\min ^{-1}$ to 0.3 events $\mathrm{min}^{-1}$. We therefore conclude that XMAP215 antagonizes the activity of XKCM1, both in interphase and in mitosis.

XMAP215 is required for spindle assembly. As changes in microtubule dynamics are essential for spindle assembly, and because XMAP215 is a major regulator of microtubule dynamics, we wondered how removal of XMAP215 would affect formation of the mitotic spindle. We added sperm nuclei to mitotic, XMAP215depleted extracts and monitored the formation of bipolar spindles $20 \mathrm{~min}$ and $45 \mathrm{~min}$ after addition of sperm nuclei. At $20 \mathrm{~min}$, we observed short microtubule asters around chromatin in XMAP215depleted extracts. The length of microtubules depended on the
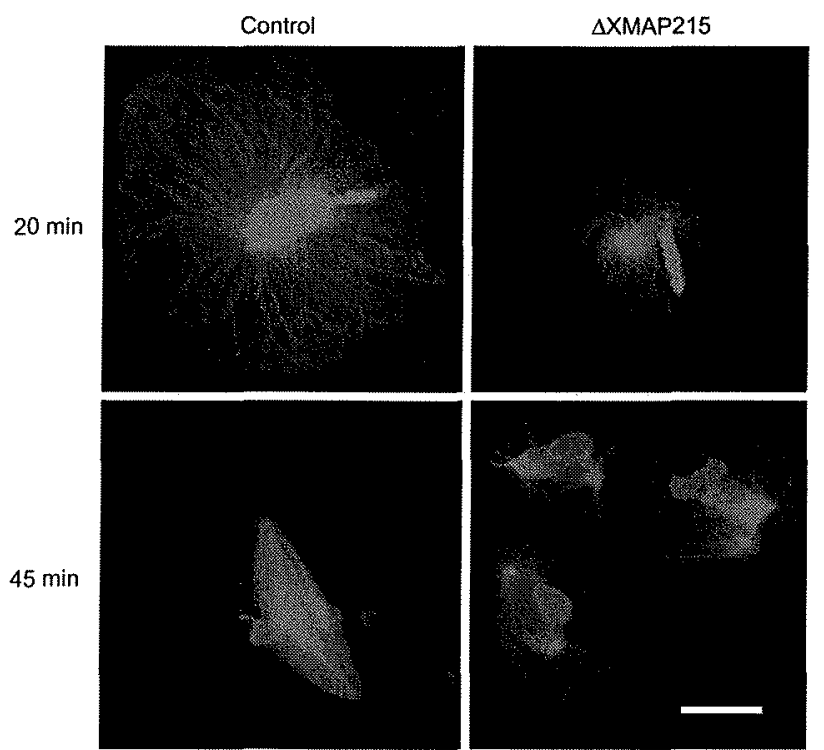

Figure 7 Spindle formation observed in control and XMAP215-depleted extracts. Microtubule arrays are seen in control (left) and XMAP215-depleted (right) extracts 20 and $45 \mathrm{~min}$ after addition of sperm nuclei. Spindles are seen $45 \mathrm{~min}$ after sperm addition. Scale bar represents $10 \mu \mathrm{m}$. depletion efficiency. When the depletion was close to $90 \%$, no microtubules were seen growing from the centrosome. In extracts from which $60 \%$ of XMAP215 was removed, microtubules were $\sim 10 \mu \mathrm{m}$ long (Fig. 7) compared with $\sim 25-\mu \mathrm{m}$-long control microtubules. $45 \mathrm{~min}$ after addition of sperm nuclei to control extracts, normal bipolar spindles of width $31.8 \pm 4.9 \mu \mathrm{m}(n=32)$ were visible. In XMAP215-depleted extracts, short spindle-like structures of width $18.3 \pm 3.9 \mu \mathrm{m}(n=33)$, containing a few short microtubules and monopolar spindles, were formed (Fig. 7). With higher depletion efficiency, we observed either very short or no microtubules nucleated by the sperm centrosome. We therefore concluded that XMAP215 is required for efficient spindle assembly.

\section{Discussion}

XMAP215 is a member of a family of proteins that have been conserved in yeasts, C. elegans, Drosophila, plants and humans. The main regions of homologies representing functional conservation are in their $\mathrm{N}$ termini ${ }^{16,17}$. Mutations in the C. elegans $z y g-9$ gene ${ }^{17}$ have effects on microtubule organization that are similar to those produced by depletion of XMAP215 from Xenopus egg extracts: microtubules are short and spindle assembly is compromised. We have shown that, in interphase, XMAP21 5 localizes along the length of microtubules and concentrates at centrosomes. This localization is similar to that of $p 93^{\text {Dist }}$, the $S$. pombe XMAP215 homologue ${ }^{19-21}$, but different from that of ZYG-9 (ref. 17) and Mini spindle proteins ${ }^{16}$, which localize primarily to centrosomes during both interphase and mitosis. It is possible that XMAP215 has more than one function in regulating the microtubule network. It may regulate microtubule stability on the one hand and microtubule nucleation on the other, an idea supported by analysis of the role of XMAP215 in Ran-GTP-dependent microtubule nucleation ${ }^{31}$.

To determine the function of XMAP215 in the context of other regulators of microtubule dynamics, we depleted the protein from Xenopus egg extracts. After depletion of XMAP215 from interphase extracts, the average growth rate of microtubules dropped from $14.2 \mu \mathrm{m} \mathrm{min}^{-1}$ to $8.0 \mu \mathrm{m} \mathrm{min} \mathrm{m}^{-1}$. This result supports the previous work of Gard and Kirschner ${ }^{14}$, who showed that XMAP215 is the major activity stimulating microtubule growth that can be purified from a Xenopus egg extract. At the concentration of tubulin present in the extracts $(20 \mu \mathrm{M})$, microtubules are expected to grow at a rate of $2.0 \mu \mathrm{m} \mathrm{min}^{-1}$ if no other factors are present ${ }^{1,2}$. Thus the residual growth rate of $6.0 \mu \mathrm{m} \mathrm{min}^{-1}$ indicates that factors that can stimulate microtubule growth are still present following XMAP215 depletion. 
One possibility is that, as we depleted only about $60 \%$ of the protein in our experiments, the residual XMAP215 stimulates growth. Alternatively, other factors, such as XMAP230 (refs 7, 32), can stimulate growth in extracts.

Perhaps the most striking effect of XMAP215 depletion from interphase extracts is the dramatic increase in the catastrophe frequency. At first glance, the activity of XMAP215 appears to be different in different circumstances. On addition to purified tubulin XMAP215 stimulates the growth and shrinkage rates without suppressing catastrophes ${ }^{5,6}$. In this system, catastrophes are rare and are due to the inherent properties of the tubulin polymer. Clearly, XMAP215 cannot suppress these types of catastrophe. However Xenopus in egg extracts, XMAP215 both stimulates growth and shrinkage rates and suppresses catastrophes by opposing the activity of XKCM1. It is possible that XKCM1 and XMAP215 compet directly for microtubule ends. Studies of the activity of XKCMI have shown that it acts on microtubule ends to stimulate microtubule depolymerization $^{12}$. Although there is no direct evidence that XMAP215 binds to microtubule ends, early studies of XMAP215 showed that it could stimulate microtubule growth at substoichiometric levels and specifically at plus ends $s^{5,6,14}$. The idea that protein bind to microtubule ends to modulate their dynamics is attractive and is further supported by work with CLIP-170, which localizes to growing microtubule ends $s^{33-35}$, and by the ability of kinetochores to distinguish ends of microtubules from the lattice ${ }^{36}$.

These results have important consequences for understanding the control of microtubule turnover between interphase and mitosis. Our observations show that XKCMI is active in mitosis as wel as in interphase. However, $\mathrm{XKCM} 1$ is prevented from stimulating catastrophes by the action of XMAP215, mainly in interphase. During mitosis, XMAP215 is phosphorylated and less able to promote microtubule growth, although it is still able to bind to microtubules ${ }^{5,6,14}$. Thus, one likely model that can explain the increase in turnover of microtubules between interphase and mitosis is that XMAP215 promotes microtubule growth in interphase, thus exerting a dominant microtubule-stabilizing activity over the microtubule-destabilizing activity of XKCM1. However, in mitosis, it does not stimulate microtubule growth, allowing catastrophe factors to act more effectively. This hypothesis is supported by the observation that depleting XMAP215 from interphase extracts reduces the growth rate of microtubules to that in mitotic extracts. The finding that immunodepletion of XMAP215 from mitotic extracts leads to an increase in catastrophe frequency in the absence of a change in growth rate is also consistent with this idea. This further indicates that, in mitosis, by binding to microtubules, XMAP215 can still oppose XKCMI activity to a certain extent Thus, a precise adjustment of microtubule turnover could be achieved by subtle regulation of the activity of XMAP215.

Although we are beginning to understand how microtubule dynamics are regulated during the cell cycle, we still know little about how this property is modulated to assemble a bipolar spindle. It is thus interesting that depleting XMAP215 from mitotic extracts results in a shorter steady-state length of microtubules. The consequence of this change in microtubule dynamics is that small spindles or no spindles at all form. Similar effects on the relationship between spindle size and microtubule dynamics were seen in previous experiments in which the catastrophe rate was increased by adding Stathmin/Op18 (ref. 37). Thus, the results described here and in ref. 37 indicate that spindle length is determined in large part by the dynamic properties of microtubules, and may be achieved mainly by regulating the frequency of catastrophes, as predicted by a mathematical model that links microtubule dynamics to their average steady-state length ${ }^{38}$

\section{Methods}

cloning.

Mass spectroscopy results (A. Podtelezhnikov et al, personal communication) indicated that XMAP215 was highly homologous to the human protein ch-TOG'. We therefore screened a $X$. laevis $00 \mathrm{cyte} \mathrm{CDNA}$ library (courtesy of . Shuttleworth) using a clone coding for KIAA0097, obtained from T. Nagase (GenBank accession number $\mathrm{D} 43948$ ), Positive clones contained either a 4.7-kilobase (kb) $3^{\prime}$-terminal part of a gene homologous to $\mathrm{ch}$-roG, or a $2-\mathrm{kb} 5^{\prime}$-terminal part that partially overlapped with the above $4.7-\mathrm{kb}$ clone but lacked the translation initiation codon. The most $5^{\prime}$ end of the gene was recovered usin $5^{\prime}$ RACE (rapid amplification of cloned ends) s. $^{30}$ with semi-nested primers (primer 1: 5'-GCCATRGACCACTTC; primer 2: $5^{\prime}$-AATRCCTGCCACGTGAGCAT). Southern hybridization with the oh-TOG probe highlighted a band of $\sim 400$ base pairs (bp), which was subcloned and sequenced to reveal high homology to the corresponding $5^{\prime}$ terminus of the ch-TOG gene. This 381 -bp fragment was used to reconstitute the $5^{\prime}$ end of the gene on the $2-\mathrm{kb} \mathrm{cDNA}$ clone. Next, a single nucleotide replacement was introduced by five cycles of Pfi- $\mathrm{PCR}$ (polymerase chain reaction) into the 28-bp region of the overlap between two $\mathrm{cDNAs}$. This mutation was silent and created a unique Dral restriction site. Finally, to assemble the whole XMAP215 gene, we carried out a three-way ligation between two cDNA clones $\{2.1$ $\mathrm{kb}$ and $4.7 \mathrm{~kb}$, cut out using Ncol $+\mathrm{Dral}$ and $\mathrm{Dral}+\mathrm{AvrlI}$, respectively) and pGEM-T (Promega), linearized with $\mathrm{NcoI}+$ Spel.

\section{Indirect immunofluorescence microscopy.}

XL177 cells were grown on $12-\mathrm{mm}$ coverslips and fixed in 3-4\% paraformaldehyde and $0.1 \%$ glutaraldehyde plus $0.5 \%$ Triton $X-100$ in BRB 80 buffer $(80 \mathrm{mM}$ PIPES, $3 \mathrm{mM} \mathrm{MgCl}, 1 \mathrm{mM}$ EGTA, $\mathrm{pH}$ 6.8). Free aldehydes were quenched by incubation in $0.1 \% \mathrm{NaBH}_{4}$ in PBS. Cells were blocked in PBS plus $0.2 \%$ Tween-20 supplemented with $10 \%$ fetal calf serum and then incubated with a $1: 200$ dilution of affuity-purified rabbit antibody towards the $N$ terminus of XMAP215 and a 1:200 dilution of mouse monoclonal anti- $\alpha$-tubulin antibodies (Amersham). Anti-mouse IgG coupled to Alexa-488 (Molecular Probes; 1:1,000 dilution) and anti-rabbit lgG coupled to $\mathrm{Cy} 3$ (Amersham; 1:500 dilution) were used as secondary antibodies. Stained cells were preincubated in $2 \mu \mathrm{g} \mathrm{ml}^{-1}$ Hoechst 3352 to stain the DNA

washed and mounted in the ProLong mounting medium (Molecular Probes). Analysis was done with an LSM 510 confocal microscope (Zeiss). The best localization was obtained using the antibodies towards the $\mathrm{N}$ termini of XMAP215 or ch-TOG. Similar localization of nicrotubules in interphase was observed in interphase microtubule asters assembled in Xenopus egg extracts (A. P., unpublished observations).

\section{Antibody production.}

The first 214 and the last 328 amino acids of KIA0097/ch-TOG were fused to glutathione-S-transferase (GST) in the pGEX-4T vector (Pharmacia) and the recombinant proteins were purified and used to obtain rabbit polyclonal antibodies. Antibodies were affinity-purified according to standard protocolst" To remove antibodies towards GST, we passed the affinity-puriffed antibodies over a GST col umn. Antibodies to XMAP215 were raised against the first 573 and the last 899 amino acids, fused to a His, tag and affinity-purified against the same fusion proteins.

\section{Preparation of Xenopus egg extracts and depletion of XMAP215.}

Xenopus egg extracts were prepared as described, and spindle assembly and microtubule dynamics wer assayed as described ${ }^{\text {sis } 1,22}$. Depletion of XMAP215 and treatment of the immunoprecipitates were done as in ref. 10 using $200 \mu \mathrm{g}$ anti-ch-TOG antibody coupled to $50 \mu \mathrm{l}$ Biorad Affi-Prep beads. Depleted extracts were used to assay spindle assembly or microtubule dynamics. XKCM1 inhibition was achieved using antibodies raised against the $\mathrm{N}$-terminal part of XKCM1 (ref. 10). Antibodies were added to the extract at a final concentration of $170 \mu \mathrm{gml}^{-3}$ before recording microtubule dynamics. Chromopure normal rabbit IgG (Dianova) was used as a control antibody in amounts equal to that of anti-TOG antibodies. To stimate the depletion efficiency of XMAP215, we loaded $1 \mu 1,0.3 \mu \mathrm{l}$ and $0.1 \mu \mathrm{l}$ of control depleted extracts, and $1 \mu \mathrm{l}$ of XMAP215-depleted extracts, onto SDS gel. The extent of the depletion was deduced from the relative intensity of the XMAP215 band, revealed by immunoblotting using $0.14 \mathrm{~g} \mathrm{ml}^{-2}$ of antibody to the $\mathrm{ch}-\mathrm{TOG}$ C terminus.

To confirm the specificity of the ch-TOG antibodies, we performed a depletion using antibodies directed against the $C$ terminus of XMAP215. These antibodies also retrieved a single protein of $M_{\mathrm{r}} 215 \mathrm{~K}$ as observed by Coomassie blue staining (data not shown). Moreover, the anti-ch-TOG antibodies recognized the $215 \mathrm{~K}$ band immunoprecipitated by the anti-XMAP215 antibody, as determined by immunobloting (data not shown)

Microtubule dynamics was recorded as described ${ }^{\text {te }}$ with the following modifications. Recording was normally arbitrarily stopped after $5 \mathrm{~min}$. However, in XMAP215-depleted extracts it was necessary to record for up to $15 \mathrm{~min}$. In one XMAP215-depleted extract in which the depletion efficiency was $90 \%$ we were unable to observe any microtubules even after $30 \mathrm{~min}$ of observation. We analysed 128, 106, 91 and 74 microt tbules, undergoing $19,79,61$ and 76 catastrophes, respectively, for the interphase control depleted, interphase XMAP215-depleted, mitotic control depleted and mitotic XMAP215-depleted extracts, respectively.

\section{Purification of XMAP215 and add-back experiments.}

Xenopus XMAP215 was purified by immunoaffinity chromatography as described". Antibodies towards a peptide from the XMAP215 $\mathrm{C}$ terminus were generated against the synthetic peptide (C)DDLKKRLERIKSSRK (synthesized by Genosis, UK), corresponding to the last 15 amino acids of XMAP215. $100 \mu$ I Affi-Prep beads (Biorad) were coated with $50 \mu \mathrm{g}$ affinity-purified anti-C-terminalpeptide antibody by incubation for $\mathrm{I} h$ at $4^{\circ} \mathrm{C}$. Beads were then washed twice with PBS- $\mathrm{T}$ ( $\mathrm{PBS}+0.1 \%$ Triton X-100) and then three times with XB-PIs (XB buffer containing $10 \mu \mathrm{gm}^{-1}$ each of leupeptin, pepstatin and chymostatin). After removing as much buffer as possible, we added $5 \mathrm{ml}$ interphase egg extract to the beads and rotated them for 1 h at $4^{\circ} \mathrm{C}$. The beads were washed three times with XB-PIs and poured into a column. To elute $X M A P 215$, we added an equal volume of $X B-P 1 s$ containing $400 \mathrm{mM} \mathrm{KCl}$ and $1.2 \mathrm{mM}$ XMAP215 $\mathrm{C}$-terminal peptides to the resin. After elution, $0.01 \% \mathrm{BSA}$ was added as a carrier protein. In controls we used normal rabbit IgG (Dianova), and we treated the controls as above.

To produce recombinant XMAP215, we used the Bac-to-bac baculovirus expressien system according to the manufacturer's instructions (Gibco). The full-length XMAP215 gene was cloned into a pFastBac vector. His insect cells were then infected to express the recombinant protein. Cells were collected and resuspended in lysis buffer $(20 \mathrm{mM}$ cation buffer, pH 7.5 (consisting of $6.7 \mathrm{mM}$ each of sodium acetate, MES and HEPES) containing $5 \%$ glycerol, $50 \mathrm{mM} \mathrm{NaCl}, 10 \mu \mathrm{gml}^{-1}$ protease inhibitor (leupeptin, pepstatin and chymostatin), $0.1 \%$ Triton $\mathrm{X}-100,10 \mathrm{mMCaCl}$ and $10 \mu \mathrm{gml}^{-3}$ nocodazole). Cells were broken using a Dounce homogenizer with 25 strokes and put on ice for $20 \mathrm{~min}$. After adding $1 \mathrm{mM}$ dithiothreitol, we centrifuged cell extracts for $10 \mathrm{~min}$ at $4^{\circ} \mathrm{C}$ at 90,000 r.p.m. on a Beckman 
TLA100.2 fotor. The supernatant was loaded onto a Mono-S column (Pharmacia) and washed with lysis buffer containing $100 \mathrm{mM} \mathrm{NaCl}$. XMAP2 15 was eluted with a $100-600 \mathrm{mM} \mathrm{NaCl}$ gradient in lysis buffer XMAP215 eluted at $\sim 400 \mathrm{mM} \mathrm{NaCl}$. The peak fractions were pooled and concentrated 50 -fold by Microcon-30 concentrators (Amicon).

In add-back experiments, a $1 / 10$ volume of purified proteins was added to XMAP215-depleted interphase extracts before recording microtubule dynamics. Elution fractions of the IgG immunoaffinit column and lysis buffer were used as controls for add-back of Xenopus XMAP215 and recombinan XMAP215, respectively. RECEIVED I6 JULY 1999; REVISED 19 OCTOBER 1999; ACCEPTED 12 NOVEMBER 1999;
PUBLISHED 29 NOVIMMBR 1999.

1. Mitchison, T. \& Kirschner, M. Dynamic instability of microtubule growth. Nature 312, 237-242 (1984)

2. Desai, A. \& Mitchison, T. I, Microtubule polymerization dynamics. Amm. Rev. Cell. Dev. Biol. 13,83117 (1997).

3. Walker, R. A. et al. Dynamic instability of individual microtubules analyzed by video light microscopy: rate constants and transition frequencies $J$ Cell Biol. 107, 1437-1448 (1988).

4. Cassimeris, L. Accessory protein regulation of microtubule dynamics throughout the cell cycle. Curr. Opin. Cell Biol, 11, 134-141 (1999).

5. Vasquez, R. J., Gard, D. L. \& Cassimeris, L. XMAP from Xenopus eggs promotes rapid plus end assembly of microtubules and rapid microtubule polymer turnover. f. Cell Biol. 127, 985-993 (1994)

6. Vasquez, R. I., Gard, D. L. \& Cassimeris, L. Phosphorylation by CDK1 regulates XMAP215 function in vitro. Cell Motil Cyloskel, 43, 310-321 (1999).

7. Andersen, S. S., Buendia, B., Dominguez, J. E., Sawyer, A. \& Karsenti, E. Effect on microtubule dynamics of XMAP230, a microtubule-associated protein present in Xenopus laevis eggs and dividing cells. J. Cell Biol. 127, 1289-1299 (1994).

8. Andersen, S. S. \& Karsenti, E. XMAP310: a Xenopus rescue-promoting factor localized to the mitotic spindle. J. Cell Biol. 139, 975-983 (1997).

9. Belmont, L. D. \& Mitchison, T. J. Identification of a protein that interacts with tubulin dimers and increases the catastrophe rate of microtubules. Cell 84, 623-631 (1996).

10. Walczak, C. E., Mitchison, T. J. \& Desai, A. XKCM1: a Xenopus kinesin-related protein that regulate microtubule dynamics during mitotic spindle assembly. Cell 84, 37-47 (1996).

11. Curmi, P. A. et al. The stathmin/tubulin interaction in vitro. J. Biol. Chem. 272, 25029-25036(1997)

12. Desai, A., Verma, S., Mitchison, T. J. \& Walczak, C. E. Kin I kinesins are microtubule-destabilizing enzymes. Cell 96, 69-78 (1999).

13. Howell, B., Larsson, N., Gullberg, M. \& Cassimeris, L. Dissociation of the tubulinusequestering and unicrotubule catastrophe-promoting activities of oncoprotein 18/stathmin. Mol. Biol. Cell 10, 105118 (1999).

14. Gard, D. L. \& Kirschner, M. W. A microtubule-associated protein from Xetopus eggs that specifically promotes assembly at the plus-end. J. Cell Biol. 105, 2203-2215 (1987).

15. Charrasse, S. et al. The ToGp protein is a new human microtubule-associated protein homologous to the Xenopus XMAP215. J. Cell Sci. 111, 1371-1383 (1998).

16. Fïona Cullen, C., Deák, P., Glover, D. M. \& Ohkura, H. mini spindles: a gene encoding a conserved microtubule-associated protein required for the integrity of the mitotic spindle in Drosophila. J. Cell Biol. 146, 1005-1018 (1999).

17. Matthews, L. R., Carter, P., Thierry-Mieg, D. \& Kemphues, K. ZYG-9, a Caenorhabditis elegans protein required for microtubule organization and function, is a component of meiotic and mitotic spindle poles. J. Cell Biol. 141, 1159-1168 (1998).

18. Wang, P. J. \& Huffaker, T. C. Stuzp: a microtubule-binding protein that is an essential component of the yeast spindle pole body. J. Cell Biol. 139, 1271-1280 (1997)

19. Nabeshima, K. et al. p93dis1, which is required for sister chromatid separation, is a novel microtubul and spindle pole body-associating protein phosphorylated at the $\mathrm{Cdc} 2$ target sites. Gentes $\mathrm{Dev} .9$, $1572-1585$ (1995)

20. Nakaseko, Y., Nabeshima, K., Kinoshita, K. \& Yanagida, M. Dissection of fission yeast microtubule associating protein p93Dis1: regions implicated in regulated localization and microtubule interaction. Genes Cells 1, 633-644 (1996)

21. Ohkura, H. et ah Cold-sensitive and caffeine-supersensitive mutants of the Schizosaccharomyces pombe dis genes implicated in sister chromatid separation during mitosis. EMBO I. 7, 1465-1473 (1988).

22. Charrasse, S. et al. Characterization of the CDNA and pattern of expression of a new gene overexpressed in human hepatomas and colonic tumors. Eur. J. Biochem. 234, 406-413 (1995).
23. Nagase, T. ef al. Prediction of the coding sequences of unidentified human genes. Ill. The coding sequences of 40 new genes (KIAA0081-KIAA0120) deduced by analysis of cDNA clones from human cell line KG-1. DNA Res. 2, 37-43 (1995).

24. Andrade, M. A. \& Bork, P. HEAT repeats in the Huntington's disease protein. Nat ure Genef. 11, 115 116 (1995).

25. Drewes, G., Ebneth, A., Preuss, U., Mandelkow, E. M. \& Mandelkow, E. MARK, a novel fanily of protein kinases that phosphorylate microtubule-associated proteins and trigger microttbule disruption. Cell 89, 297-308 (1997)

26. Aizawa, $\mathrm{H}$. el al. Molecular cloning of a ubiquitously distributed microtubule-associated protein with Mr 190,000. J. Biol. Chem. 265, 13849-13855 (1990).

27. Chapin, S. J. \& Bulinski, J. C. Non-neuronal $210 \times 10(3)$ Mr microtubule-associated protein (MAP4) contains a domain homologous to the microtubule-binding domains of neuronal MAP2 and tau. /. Cell Sci. 98, 27-36 (1991)

28. Goedert, M., Wischik, C. M. Crowther, R. A., Walker, J. E. \& Klug, A. Cloning and sequencing of the CDNA encoding a core protein of the paired helical filament of Alzheimer disease: identification as the microtubule-associated protein tat. Proc. Natl Acad. Sci. USA 85, $4051-4055$ (1988).

29. West, R. R., Tenbarge, K. M. \& Olmsted, J. B. A model for microtubule-associated protein 4 structure, Domains defined by comparisons of human, mouse, and bovine sequences. J. Biol. Chem. 266 21886-21896 (1991).

30. Tournebize, R. et al. Distinct roles of PP1 and PP2A-like phosphatases in control of microtubule dynamics during mitosis. EMBO J. 16, 5537-5549 (1997)

31. Wilde, A. \& Zheng, Y. Stimulation of microtubule aster formation and spindle assembly by the smal GTPase Ran, Science 284, 1359-1362 (1999)

32. Shiina, N., Moriguchi, T., Ohta, K., Gotoh, Y. \& Nishida, E. Regulation of a major microtubuleassociated protein by MPF and MAP kinase. EMBO I. 11, 3977-3984 (1992).

33. Diamantopoulos, G. S. et al. Dynamic localization of CLlP-170 to microt ubule plus ends is coupled to microtubule assembly. I. Cell Biol. 144, 99-112 (1999).

34. Perez, F., Diamantopoulos, G. S., Stalder, R. \& Kreis, T. E. CLIP-170 highlights growing microtubule ends in vivo. Cell 96, 517-527 (1999).

35. Vaughan, K. T., Tynan, S. H., Faulkner, N. E., Echeverri, C. I. \& Vallee, R. B. Colocalization of cytoplasmic dynein with dynactin and CLIP-170 at microtubule distal ends. I. Cell Sci. 112, 1437$1447(1999)$.

36. Severin, F. F., Sorger, P. K. \& Hyman, A. A. Kinetochores distinguish GTP from GDP forms of the microtubule lattice. Nature 388, 888-891 (1997)

37. Andersen, S. S. et al. Mitotic chromatin regulates phosphorylation of Stathmin/Op18. Nature 389, 640-643 (1997).

38. Verde, F., Dogterom, M., Stelzer, E., Karsenti, E. \& Leibler, S. Control of microtubule dynamics and length by cyclin A- and cyclin B- dependent kinases in Xenopus egg extracts. J. Cell Biol. 118, 1097 1108 (1992).

39. Frohman, M. A., Dush, M. K. \& Martin, G. R. Rapid production of full-length cDNAs from rare transcripts: amplification using a single gene-specific oligonudeotide primer. Proc. Natl Acad. Sci USA 85, 8998-9002 (1988).

40. Harlow, E. \& Lane, D. Amibodies ; a Laboratory Mamaal (Cold Spring Harb. Lab. Press, Cold Spring Harbor, 1988).

41. Murray, A. W. in Xenopus lnevis: Practical Uses in Cell and Molecular Biology (eds Kay, B. K. \& Peng H. B.) $581-605$ (Academic, San Diego, 1991).

42. Sawin, K. E. \& Mitchison, T. J. Mitotic spindle assembly by two different pathways in vitro. J. Cell biol $112,925-940$ (1991).

43. Field, C. M., Oegema, K., Zheng, Y., Mitchison, T. ) \& Walczak, C. E. Purification of cytoskeletal proteins using peptide antibodies. Methods Enzymol. 298, 525-541 (1998).

ACKNOWLEDGEMENTS

We thank F. Senger for cell culture; $D$. Drechsel for help during protein purification; A. Shevchenko and A. Shevchenko for mass spectrometry analysis; A. Desai for stimulating discussions and advice; and A. Desai, 1. Sassoon, P. Gönczy, K. Oegema, M. Rathman, M. Mavris and C. Gonzalez for critical reading of the manuscript. A.P. was supported by an EMBO long-term and an HSFPO long-term fellowship. K.K. was supported by an HFSPO long-term fellowship.

Correspondence and requests for materials should be addressed to A.A.H. The XMAP215 cDNA sequence has been submitted to EMBL Nudeotide Sequence Database under accession number Aj251130. 